\title{
Identification of Risk Factors Associated With Stage III Disease in Nonmetastatic Colon Cancer: Results From a Prospective National Cohort Study
}

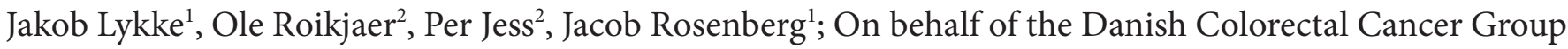 \\ ${ }^{1}$ Department of Surgery, Herlev Hospital, University of Copenhagen, Herlev; ${ }^{2}$ Department of Surgery, Zealand University Hospital, Roskilde, \\ Denmark
}

Purpose: This study aimed to identify possible patient- and tumor-related factors associated with risk of TNM stage III disease in nonmetastatic colon cancer.

Methods: The associations between stage III disease and age, sex, lymph node yield, pathological tumor (pT) stage, tumor subsite, type of surgery, and priority of surgery were assessed in a nationwide cohort of 13,766 patients treated with curative resection of colon cancer. Each level of age, lymph node yield, and pT stage was compared to the preceding level. Results: Age, lymph node yield, pT stage, tumor subsite, and priority of surgery were associated with stage III disease. Odds ratios (95\% confidence interval [CI]) were as follows: age <65/65-75 years: 1.28 (95\% CI, 1.15-1.43) and 6575/ > 75 years: 1.22 (95\% CI, 1.13-1.32); lymph node yield 0-5/6-11: 0.60 (95\% CI, 0.50-0.72), lymph node yield 6-11/12-17: 0.84 (95\% CI, 0.76-0.93), and lymph node yield 12-17/ $\geq 18: 0.97$ (95\% CI, 0.89-1.05); pT1/pT2: 0.74 (95\% CI, 0.57-0.95), pT2/pT3: 0.35 (95\% CI, 0.30-0.40), and pT3/pT4: 0.49 (95\% CI, 0.47-0.54). Only tumors of the transverse colon were independently associated with lower risk of stage III disease than tumors in the sigmoid colon (sigmoid colon: 1, transverse colon: 0.84 [95\% CI, 0.73-0.96]; elective surgery: 1, acute surgery: 1.43 [95\% CI, 1.29-1.60]).

Conclusion: In this study, stage III disease in colon cancer was significantly associated with age, lymph node yield, pT stage, tumor subsite, and priority of surgery but was not associated with right-sided location compared with stage I and II cancers.

Keywords: Colonic neoplasms; Lymph nodes; Neoplasm staging; Tumor subsite

\section{INTRODUCTION}

Colon cancer is one of the most common types of cancers and one of the primary causes of cancer death [1].

Surgery is the main treatment for colon cancer. The primary determinants of prognosis are presence of distance metastasis and pathological tumor (pT) stage including local lymph node in-

Received: Jan 20, 2019 - Revised: Feb 21, 2019 - Accepted: Mar 3, 2019 Correspondence to: Jakob Lykke, Ph.D.

Department of Surgery, Herlev Hospital, Herlev Ringvej 75, 2730 Herlev, Denmark

Tel: +45-2624-0898

E-mail: jaklyk@gmail.com

ORCID: https://orcid.org/0000-0001-9311-9964

(C) 2020 The Korean Society of Coloproctology

This is an open-access article distributed under the terms of the Creative Commons Attribution NonCommercial License (https://creativecommons.org/licenses/by-nc/4.0) which permits unrestricted non-

commercial use, distribution, and reproduction in any medium, provided the original work is properly cited. volvement. The most widely used staging system is the TNM classification of malignant tumors. Many of its features have been considered by the American Joint Committee on Cancer (AJCC)/ the Union for International Cancer Control (UICC) $[2,3]$, although its validity has been debated [4-6]. The AJCC/UICC guidelines for colon cancer are founded on more than 20-year-old recommendations based on level III to IV evidence [7]. These recommendations concluded that, to achieve an accuracy of $>90 \%$, a minimum lymph node yield of 12 negative lymph nodes is needed to guarantee lymph node-negative disease in colon cancer [7]. Despite these guidelines, debate remains regarding the optimal lymph node yield to guarantee proper staging $[5,6]$. Moreover, subsequent studies have demonstrated that patient-related and histopathological factors including sex, age, tumor location, body mass index, $\mathrm{pT}$ category, and elective vs. acute surgery seem to influence the risk of stage III disease in colon cancer [8-12]. Although most countries have accepted the guideline of 12 lymph 
nodes, recent national cohorts fulfill the 12 lymph node recommendation in only a limited number of patients $[11,13,14]$.

The overall aim of this study in a high-quality national cohort with nonmetastatic colon cancer was to identify possible patientand tumor-related factors associated with risk of stage III disease.

\section{METHODS}

Since May 2001, when the Danish Colorectal Cancer Group (DCCG) established a nationwide database, all patients aged 18 years or older with a first-time diagnosis of colorectal adenocarcinoma treated in any Danish colorectal surgical department have been prospectively recorded. Patients are identified by unique civil registry numbers, which have been allocated by the Central Population Registry to all Danish citizens since 1968. Data are provided by surgical departments and include demographics, tumor location, tumor category, diagnostic and therapeutic procedures, and postoperative complications. The completeness of data collection in the DCCG database is estimated annually and has improved from $86.4 \%$ to $96.7 \%$ in the study period of 2001 to 2011 [15]. The high completeness is achieved through linkage to the Danish National Patient Register.

In our study cohort, the DCCG database and the Danish Pathology Registry were used for data extraction and analyses. Histopathology of the primary tumor was extracted from the Danish Pathology Registry. Variables of age, sex, tumor location, pT category, $\mathrm{N}$ category, open vs. laparoscopic surgery, acute vs. elective surgery, and lymph node yield were extracted for use in the present study. The Dukes classification was standard for staging patients with colorectal cancer in Denmark in the first 2 years of the database (2001 to 2002) [15]. Since this method does not specify pT category, patients from that period were excluded. Only patients with a first-time diagnosis of colon cancer and subsequent curative colon resection, defined as R0-resection of stage I to III adenocarcinoma located at least $15 \mathrm{~cm}$ beyond the anal verge, were included. Data from Jan 1, 2003 to December 31, 2011 were included.

The study was approved by the Danish Data Protection Agency (No. 2000-53-0073). Data were accessed and analyzed on secured servers from Denmark's Statistics. Due to Danish legislation, informed consent is not needed in a register-based research project with anonymized data [16]. In Denmark, all patients with colon cancer are treated in public hospitals.

The risk of stage III disease was analyzed according to sex, priority of surgery, type of surgery, 3 groups defined by age, 4 groups determined by lymph node yield, and tumor location. Colon cancer localization was described according to site; right-sided colon cancer (cecum, ascending colon, hepatic flexure, and transverse colon) and left-sided colon cancer (from splenic flexure to sigmoid colon, both included) and to specific subsites (cecum, ascending colon, hepatic flexure, transverse colon, splenic flexure, descending colon, and sigmoid colon). The border between the sigmoid colon and rectum was defined as $15 \mathrm{~cm}$ from the anal verge.

The associations between stage III disease and year of diagnosis, pT category, age (3 groups), lymph node yield (4 groups), sex, open vs. laparoscopic surgery, acute vs. elective surgery, rightsided vs. left-sided colon cancer, and tumor subsites were explored using multiple logistic regressions with lymph node positivity as an independent variable. Data was reported as odds ratio (OR) with 95\% confidence interval (95\% CI). For age, lymph node yield, and pT category, each level of the variable was compared with the preceding level.

Data were analyzed using IBM SPSS ver. 22.0 (IBM Corp., Armonk, NY, USA). Patient characteristics and tumor-related data were analyzed by Pearson chi-square test. Data are described as median and interquartile range (IQR). A P-value of $<0.05$ was used as the level of significance in all analyses.

\section{RESULTS}

\section{Patient characteristics}

Data from 13,766 patients (male, 48.7\%) with radical resection of stage I to III colon cancer were available for the analysis (Fig. 1). The median age was 70 years (IQR, 62 to 78 years), and median lymph node yield was 15 (IQR, 11 to 22 ). A total of $37.5 \%$ of patients had stage III disease (Table 1).

18,652 Patients with an intended curative resection of a colon cancer (UICC stage I-III) in the nationwide DCCG database assessed for inclusion

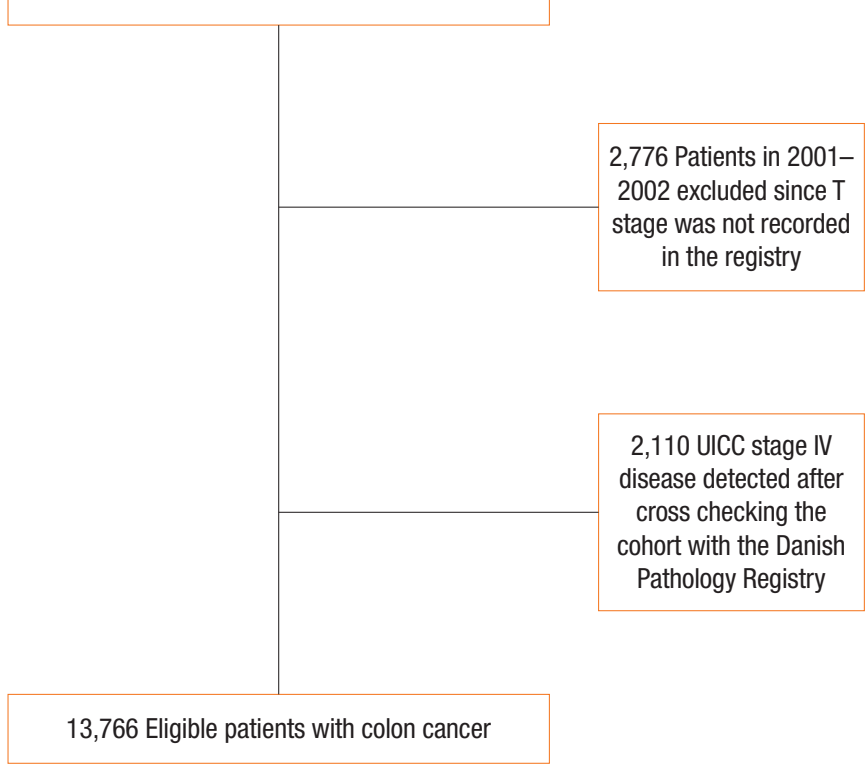

Fig. 1. The complete patient cohort indicating those groups excluded during the process to isolate the final group of patients included in the analysis. UICC, Union for International Cancer Control; DCCG, Danish Colorectal Cancer Group. 


\section{$\begin{array}{rlr}\text { Annals of } & \text { Results From a Pros } \\ \text { Coloproctology } & \text { Jakob Lykke, et al. }\end{array}$}

Identification of Risk Factors Associated With Stage III Disease in Nonmetastatic Colon Cancer:

Table 1. Patient and tumor characteristics $(n=13,766)$

\begin{tabular}{|c|c|c|c|}
\hline Characteristic & Data $^{a}$ & $\begin{array}{c}\text { Patients with lymph node-positive disease/ } \\
\text { total number of patients }(\%)\end{array}$ & P-value \\
\hline Sex & & & 0.760 \\
\hline Male & 6,694 (48.6) & 2,659/7,072 (37.6) & \\
\hline Female & $7,072(51.4)$ & 2,500/6,694 (37.3) & \\
\hline \multicolumn{4}{|l|}{ Age (yr) } \\
\hline$<65$ & $2,011(14.6)$ & $882 / 2,011(43.9)$ & $<0.001$ \\
\hline $65-75$ & $6,528(47.4)$ & 2,487/6,528 (38.1) & \\
\hline$>75$ & $5,227(38.0)$ & 1,790/5,227 (34.2) & \\
\hline Tumor side & & & 0.194 \\
\hline Right & $7,265(52.8)$ & 2,538/6,674 (38.0) & \\
\hline Left & $6,501(47.2)$ & 2,621/7,092 (37.0) & \\
\hline Tumor subsite & & & 0.009 \\
\hline Cecum & 2,718 (19.7) & $1,091 / 2,718(40.1)$ & \\
\hline Ascending colon & $1,859(13.5)$ & $679 / 1,859(36.5)$ & \\
\hline Hepatic flexure & $993(7.2)$ & 365/993 (36.8) & \\
\hline Transverse colon & $1,104(8.0)$ & 403/1,104 (36.5) & \\
\hline Splenic flexure & $5,91(4.3)$ & 241/591 (40.2) & \\
\hline Descending colon & $5,80(4.2)$ & $230 / 580(39.7)$ & \\
\hline Sigmoid colon & $5,921(43.0)$ & 2,150/5,921 (36.3) & \\
\hline pT category & & & $<0.001$ \\
\hline pT1 & 728 (5.3) & $90 / 728(12.4)$ & \\
\hline pT2 & $1,566(11.4)$ & 264/1,566 (16.9) & \\
\hline pT3 & $9,078(65.9)$ & 3,480/9,078 (38.3) & \\
\hline pT4 & 2,303 (16.7) & 1,294/2,303 (56.2) & \\
\hline Priority of surgery & & & $<0.001$ \\
\hline Elective & 11,918 (86.6) & $4,264 / 11,918(35.8)$ & \\
\hline Acute & $1,845(13.4)$ & $893 / 1,845(48.8)$ & \\
\hline Unknown & $3(0.0)$ & & \\
\hline Type of surgery & & & $<0.001$ \\
\hline Open & $9,683(70.3)$ & 3,747/9,683 (38.7) & \\
\hline Laparoscopic & $4,080(29.6)$ & $1,411 / 4,080(34.6)$ & \\
\hline Unknown & $3(0.0)$ & NA & \\
\hline Lymph node yield & $15(11-22)$ & NA & $<0.001$ \\
\hline $0-5$ & $1,001(7.3)$ & 222/1,001 (22.2) & \\
\hline $6-11$ & 2,938 (21.3) & 1,004/2,938 (34.2) & \\
\hline $12-17$ & 4,439 (32.2) & 1,735/4,439 (39.1) & \\
\hline$\geq 18$ & $5,388(39.1)$ & 2,198/5,388 (40.8) & \\
\hline
\end{tabular}

avalues are presented as number (\%) or median (interquartile range). pT, pathological tumor; NA, not applicable.

\section{Univariate analyses}

No difference in percentage of stage III disease was observed according to sex (Table 1). Proportion of stage III disease decreased significantly with increasing age (Table 1). No significant difference in proportion of stage III disease was observed for rightsided vs. left-sided colon cancers (Table 1). 
Table 2. Logistic regression analysis including tumor side (left vs. right) with a dependent variable of lymph node-positive disease

\begin{tabular}{llr}
\hline Variable & $\begin{array}{c}\text { Odds ratio } \\
(95 \% \text { confidence interval) }\end{array}$ & P-value \\
\hline Male & 1 & \\
Female & $1.001(0.931-1.077)$ & 0.980 \\
Age, 65 yr vs. 65-75 yr & $1.280(1.151-1.424)$ & $<0.001^{\mathrm{a}}$ \\
Age, 65-75 yr vs. $>75$ yr & $1.220(1.126-1.321)$ & $<0.001^{\mathrm{a}}$ \\
Elective surgery & 1 & \\
Acute surgery & $1.433(1.288-1.594)$ & $<0.001$ \\
Laparoscopic surgery & 1 & \\
Open surgery & $1.060(0.968-1.160)$ & 0.206 \\
Tumor side left & 1 & \\
Tumor side right & $0.925(0.858-0.997)$ & 0.041 \\
pT1 vs. pT2 & $0.735(0.566-0.954)$ & $0.021^{\mathrm{a}}$ \\
pT2 vs. pT3 & $0.346(0.301-0.398)$ & $<0.001^{\mathrm{a}}$ \\
pT3 vs. pT4 & $0.491(0.446-0.540)$ & $<0.001^{\mathrm{a}}$ \\
Lymph node yield, 0-5 vs. 6-11 & $0.600(0.503-0.717)$ & $<0.001^{\mathrm{a}}$ \\
Lymph node yield, 6-11 vs. 12-17 & $0.838(0.755-0.930)$ & $<0.001^{\mathrm{a}}$ \\
Lymph node yield, 12-17 vs. $\geq 18$ & $0.965(0.885-1.053)$ & $0.427^{\mathrm{a}}$ \\
Year of diagnosis & $0.962(0.946-0.979)$ & $<0.001$ \\
\hline PT, & & \\
\hline
\end{tabular}

pT, pathological tumor category.

aEach level compared to preceding level.

Tumors in the cecum, splenic flexure, and descending colon had the highest risk of stage III disease, with $39.7 \%$ to $40.2 \%$ of cases having stage III disease. Tumors in the ascending colon, hepatic flexure, transverse colon, and sigmoid colon had a risk of stage III disease ranging from $36.5 \%$ to $36.8 \%(\mathrm{P}=0.009)$. A significant association between increasing $\mathrm{pT}$ category and stage III disease was observed over a range from $12.4 \%$ in pT1 tumors to $56.2 \%$ in pT4 tumors $(\mathrm{P}<0.001)$. A significantly different proportion of stage III disease was observed in the group of patients who underwent acute operation (48.8\%) compared to those who underwent elective operation $(35.8 \%)(\mathrm{P}<0.001)$. The proportion of patients with stage III disease was $38.7 \%$ in laparoscopic surgery compared with $34.6 \%$ in open surgery $(\mathrm{P}<0.001)$. Finally, a significant difference in percentage of stage III disease in the 4 lymph node yield groups was observed, ranging from 22.2\% (lymph node yield, 0 to 5$)$ to $40.8 \%$ (lymph node yield, $\geq 18)(\mathrm{P}<0.001)$ (Table 1).

\section{Multivariate analysis}

In multivariate logistic regression analysis, age, lymph node yield, pT category, tumor site (left vs. right), and priority of surgery were all independently associated with stage III disease. Moreover, year of diagnosis was independently associated with stage III disease, and a decline in the risk of stage III disease was observed throughout the study period. No association was found between stage III
Table 3. Logistic regression analysis including tumor subsites with a dependent variable of lymph node-positive disease

\begin{tabular}{|c|c|c|}
\hline Variable & $\begin{array}{c}\text { Odds ratio } \\
\text { (95\% confidence interval) }\end{array}$ & P-value \\
\hline Male & 1 & \\
\hline Female & $0.928(0.931-1.074)$ & 0.980 \\
\hline Age, 65 yr vs. $65-75$ yr & $1.282(1.153-1.426)$ & $<0.001^{\mathrm{a}}$ \\
\hline Age, $65-75$ yr vs. $>75$ yr & $1.220(1.127-1.322)$ & $<0.001^{\mathrm{a}}$ \\
\hline Elective surgery & 1 & \\
\hline Acute surgery & $1.430(1.285-1.591)$ & $<0.001$ \\
\hline Open surgery & 1 & \\
\hline Laparoscopic surgery & $1.051(0.959-1.151)$ & 0.286 \\
\hline Sigmoid colon & 1 & \\
\hline Caecum & $0.992(0.898-1.096)$ & $0.875^{\mathrm{b}}$ \\
\hline Ascending colon & $0.897(0.800-1.007)$ & $0.064^{b}$ \\
\hline Hepatic flexure & $0.871(0.753-1.009)$ & $0.066^{b}$ \\
\hline Transverse colon & $0.836(0.726-0.962)$ & $0.013^{b}$ \\
\hline Splenic flexure & $0.986(0.824-1.181)$ & $0.881^{b}$ \\
\hline Descending colon & $0.998(0.801-1.152)$ & $0.667^{b}$ \\
\hline pT1 vs. pT2 & $0.736(0.567-0.956)$ & $0.021^{\mathrm{a}}$ \\
\hline pT2 vs. pT3 & $0.344(0.299-0.397)$ & $<0.001^{\mathrm{a}}$ \\
\hline pT3 vs. pT4 & $0.493(0.448-0.542)$ & $<0.001^{\mathrm{a}}$ \\
\hline Lymph node yield, 0-5 vs. 6-11 & $0.602(0.504-0.719)$ & $<0.001^{\mathrm{a}}$ \\
\hline Lymph node yield, 6-11 vs. 12-17 & $0.839(0.756-0.931)$ & $<0.001^{\mathrm{a}}$ \\
\hline Lymph node yield, $12-17$ vs. $\geq 18$ & $0.963(0.882-1.050)$ & $0.393^{\mathrm{a}}$ \\
\hline Year of diagnosis & $0.963(0.947-0.979)$ & $<0.001$ \\
\hline
\end{tabular}

$\mathrm{pT}$, pathological tumor category.

${ }^{a}$ Each level compared to preceding level. ${ }^{b}$ Each colon subsite compared to sigmoid colon.

disease and either sex or type of surgery. The ORs for stage III disease are shown in Table 2.

Further multivariate analysis included tumor localization by 7 tumor subsites. In this analysis, tumors of the sigmoid colon were chosen as the reference value since such tumors were found to present the lowest risk of stage III disease in univariate analysis. In this analysis, only tumors in the transverse colon were independently associated with lower risk of stage III disease. There was no difference in risk of stage III disease between tumors in the sigmoid colon and tumors in the remaining tumor subsites (Table 3).

\section{DISCUSSION}

The aim of this study was to investigate issues concerning diagnosis of stage III disease in colon cancer. We found an association between lymph node yield from a colon cancer specimen and occurrence of stage III disease. An independent association was also found between increasing lymph node yield and probability of 
detecting stage III disease up to a lymph node yield of 12 to 17 , in accordance with present guidelines.

A declining risk for stage III disease was observed with increasing age. The reason for this association is unknown, but it has been proposed that elderly patients are under-staged as a consequence of insufficient lymph node harvest due to less extensive resection $[8,17,18]$. Nevertheless, in our multivariate analysis, increasing age was independently associated with decreasing risk of stage III disease, indicating that under-staging may only be part of the explanation. Another possible explanation is that colon tumors in elderly patients behave less aggressively than those in younger patients, as proposed by others [19]. Unfortunately, such data were not available for our analysis.

An increasing rate of stage III disease was observed with more advanced $\mathrm{pT}$ stage. This relationship remained significant in multivariate analysis. It seems reasonable that more invasive tumors are more prone to disseminate to the lymphovascular system, including lymph nodes, than less invasive tumors. This is supported by other studies $[13,20]$.

It has previously been proposed that right-sided colon cancers differ from left-sided colon cancers and more often present with stage III disease [20]. Subsequent studies have proposed that this difference depends on patient- and tumor-related characteristics [20-24]. Results from our study question these findings since no difference in rate of stage III disease was observed in the rightsided vs. left-sided colon cancers in univariate analysis. However, multivariate analysis identified a significant, although minor reduced risk of stage III disease in right-sided colon cancers compared to left-sided colon cancers.

When analyzing the percentage of cases with stage III disease according to tumor subsite, tumors in the cecum, splenic flexure, and descending colon had the highest risk of stage III disease (ranging from $39.7 \%$ to $40.2 \%$ ) compared to tumors in the ascending colon, hepatic flexure, transverse colon, and sigmoid colon (ranging from $36.5 \%$ to $36.8 \%)(\mathrm{P}=0.009)$.

These findings are supported by other studies in which the cecum and splenic flexure had the highest risk of stage III disease [20]. There are several potential explanations for our findings. Endoscopic accessibility is proposed to differ according to colon subsite, and lesions located at the flexures are especially difficult to detect [25]. Therefore, tumors at these subsites may be detected at a more advanced stage than tumors in other parts of the colon. Moreover, there is an unknown rate of incomplete colonoscopy in which parts of the right side of the colon/cecum are not fully visualized due to endoscopic difficulties and incomplete bowel preparations [25]. Therefore, a number of right-sided tumors will not be detected before they cause clinical symptoms. Other studies have shown that colonic transit time and fecal exposure time in different colon subsites are associated with risk of stage III disease [20, 26].

In a multivariate analysis, only tumors in the transverse colon had an independent reduced risk of stage III disease, although minor, compared to those in the sigmoid colon, which had the lowest risk of stage III disease in univariate analyses. These results indicate that tumor subsite alone is not independently associated with risk of stage III disease.

When acute surgery was compared to elective surgery, there was significantly more acute surgery performed in patients with stage III disease. In the multivariate analysis, this difference remained significant. The main reasons for acute surgery for colon cancer are ileus or perforation. Large bulky tumors present more often with ileus and perforations than small tumors, and it is likely that large tumors have a higher risk of disseminating to local lymph nodes than small tumors. Our findings emphasize that patients who present with acute colon cancer are high-risk patients who require a skilled surgical team to ensure sufficient resection.

A decrease in risk of stage III disease during the data collection period was observed. Thus, year of diagnosis was an independent prognostic factor for risk of stage III disease. The exact reason for the decrease in risk of stage III disease over time in Denmark is unknown. Since the year 2000, a range of national initiatives including programs for early detection of colon and rectal cancer has been launched in Denmark to improve the prognostic outcome for patients with colorectal cancer. It is likely that these initiatives, orchestrated by the DCCG, together with a uniform healthcare system managed centrally by the National Board of Health, have contributed to better detection of patients with colon cancer.

It is likely that a standardized immune score system will be an integrated part of the colon cancer staging system when this system is fully developed [27]. It is unknown if a large lymph node yield will still be required for correct diagnosis after introduction of such an immune score system.

Lymph nodes, including T-cell activated lymph nodes, are a central part of the immune system, and activated T-cells are key to elimination of cancer cells. Further research is needed to understand how removal of T-cell activated lymph nodes influences tumor-immune interactions in colon cancer. Nevertheless, it seems likely that staging based on an immune score can reduce the need for a large lymph node harvest and save some T-cell activated lymph nodes to fight cancer. A staging system based on tumorimmune interactions may allow more precise identification of low- and high-risk cancers that will increase the possibility of identifying patients who will benefit from adjuvant therapy. More precise staging will increase the opportunity for less extensive resections or even allow local resections (organ-preserving resections). This will reduce the risk of surgical complications and possible side effects of extensive resection.

The current analysis has several positive attributes in addition to the fundamental strength of its national population-based design. The study included patients from all Danish departments conducting colon surgery during the study period. It was further strengthened by an almost complete and unselected compilation of data merged from 2 different population-based national registers. Moreover, patients received uniform treatment since treat- 
ment of colon cancer in Denmark was standardized following recommendations by the DCCG.

There are some limitations to the present study. Since this is an observational study, we cannot describe causal relationships but only associations. Second, we did not have data on molecular and genetic features of tumors, denying us the ability to draw any conclusions concerning the possible association between molecular and genetic characteristics of tumors and stage III disease. Thirdly, we did not have any data on the subspecialty of surgeons, which might explain acute surgery as an independent predictor.

Despite these limitations, we analyzed a national unselected cohort with $>95 \%$ data completeness and found that the risk of stage III disease in colon cancer is associated with age, pT stage, and tumor subsite. Moreover, a minimum of 12 negative lymph nodes, as recommended in the current guidelines, is needed to ensure correct diagnosis of lymph node-negative disease. Our study did not reproduce previous findings indicating that rightsided colon cancers present with stage III disease more often than left-sided colon cancers. Finally, our study confirms that patients presenting with acute colon cancer are at high risk of stage III disease compared to those operated on in an elective setting.

Based on preoperative data of age, tumor subsite, and preoperative staging, it might be possible to tailor resection for patients with colonic cancer in the future.

\section{CONFLICT OF INTEREST}

No potential conflict of interest relevant to this article was reported.

\section{ACKNOWLEDGMENTS}

Contributions from local surgical representatives of the Danish Colorectal Cancer Group are gratefully acknowledged.

\section{REFERENCES}

1. Siegel R, Naishadham D, Jemal A. Cancer statistics, 2013. CA Cancer J Clin 2013;63:11-30.

2. Edge SB, Compton CC. The American Joint Committee on Cancer: the 7th edition of the AJCC cancer staging manual and the future of TNM. Ann Surg Oncol 2010;17:1471-4.

3. Union for International Cancer Control (UICC). What is TNM? [Internet]. Geneva: UICC; c2020 [cited 2018 May 16]. Available from: https://www.uicc.org/resources/tnm.

4. Quirke P, Williams GT, Ectors N, Ensari A, Piard F, Nagtegaal I. The future of the TNM staging system in colorectal cancer: time for a debate? Lancet Oncol 2007;8:651-7.

5. Bui L, Rempel E, Reeson D, Simunovic M. Lymph node counts, rates of positive lymph nodes, and patient survival for colon cancer surgery in Ontario, Canada: a population-based study. J Surg Oncol 2006;93:439-45.
6. Baxter NN, Ricciardi R, Simunovic M, Urbach DR, Virnig BA. An evaluation of the relationship between lymph node number and staging in $\mathrm{pT} 3$ colon cancer using population-based data. Dis Colon Rectum 2010;53:65-70.

7. Nelson H, Petrelli N, Carlin A, Couture J, Fleshman J, Guillem J, et al. Guidelines 2000 for colon and rectal cancer surgery. J Natl Cancer Inst 2001;93:583-96.

8. Tekkis PP, Smith JJ, Heriot AG, Darzi AW, Thompson MR, Stamatakis JD, et al. A national study on lymph node retrieval in resectional surgery for colorectal cancer. Dis Colon Rectum 2006; 49:1673-83.

9. Gonsalves WI, Kanuri S, Tashi T, Aldoss I, Sama A, Al-Howaidi I, et al. Clinicopathologic factors associated with lymph node retrieval in resectable colon cancer: a Veterans' Affairs Central Cancer Registry (VACCR) database analysis. J Surg Oncol 2011; 104:667-71.

10. Jess P, Hansen IO, Gamborg M, Jess T; Danish Colorectal Cancer Group. A nationwide Danish cohort study challenging the categorisation into right-sided and left-sided colon cancer. BMJ Open 2013;3:e002608.

11. Nathan H, Shore AD, Anders RA, Wick EC, Gearhart SL, Pawlik TM. Variation in lymph node assessment after colon cancer resection: patient, surgeon, pathologist, or hospital? J Gastrointest Surg 2011;15:471-9.

12. Nedrebø BS, Søreide K, Nesbakken A, Eriksen MT, Søreide JA, Kørner H, et al. Risk factors associated with poor lymph node harvest after colon cancer surgery in a national cohort. Colorectal Dis 2013;15:e301-8.

13. Baxter NN, Virnig DJ, Rothenberger DA, Morris AM, Jessurun J, Virnig BA. Lymph node evaluation in colorectal cancer patients: a population-based study. J Natl Cancer Inst 2005;97:219-25.

14. Wong SL, Ji H, Hollenbeck BK, Morris AM, Baser O, Birkmeyer JD. Hospital lymph node examination rates and survival after resection for colon cancer. JAMA 2007;298:2149-54.

15. Danish Colorectal Cancer Group (DCCG). DCCG annual reports 2001-11 [Internet]. Copenhagen: DCCG; c2020 [cited 2018 Aug 27]. Available from: http://www.dccg.dk.

16. Danish National Committee on Health Research Ethics. What to notify? [Internet]. Copenhagen: Danish National Committee on Health Research Ethics; 2018 [cited 2019 Jan 7]. Available from: http://en.nvk.dk/how-to-notify/what-to-notify.

17. Wang L, Hollenbeak CS, Stewart DB. Node yield and node involvement in young colon cancer patients: is there a difference in cancer survival based on age? J Gastrointest Surg 2010;14:135561.

18. Steele SR, Chen SL, Stojadinovic A, Nissan A, Zhu K, Peoples GE, et al. The impact of age on quality measure adherence in colon cancer. J Am Coll Surg 2011;213:95-103.

19. Goldvaser H, Purim O, Kundel Y, Shepshelovich D, Shochat T, Shemesh-Bar L, et al. Colorectal cancer in young patients: is it a distinct clinical entity? Int J Clin Oncol 2016;21:684-95.

20. Benedix F, Schmidt U, Mroczkowski P, Gastinger I, Lippert H, 


\section{Coloproctology Jakob Lykke, et al.}

Kube R, et al. Colon carcinoma: classification into right and left sided cancer or according to colonic subsite? Analysis of 29,568 patients. Eur J Surg Oncol 2011;37:134-9.

21. Bufill JA. Colorectal cancer: evidence for distinct genetic categories based on proximal or distal tumor location. Ann Intern Med 1990;113:779-88.

22. Benedix F, Meyer F, Kube R, Kropf S, Kuester D, Lippert H, et al. Influence of anatomical subsite on the incidence of microsatellite instability, and KRAS and BRAF mutation rates in patients with colon carcinoma. Pathol Res Pract 2012;208:592-7.

23. Nawa T, Kato J, Kawamoto H, Okada H, Yamamoto H, Kohno H, et al. Differences between right- and left-sided colon cancer in patient characteristics, cancer morphology and histology. J Gastroenterol Hepatol 2008;23:418-23.
24. Yamauchi M, Morikawa T, Kuchiba A, Imamura Y, Qian ZR, Nishihara R, et al. Assessment of colorectal cancer molecular features along bowel subsites challenges the conception of distinct dichotomy of proximal versus distal colorectum. Gut 2012;61: 847-54.

25. Pickhardt PJ, Nugent PA, Mysliwiec PA, Choi JR, Schindler WR. Location of adenomas missed by optical colonoscopy. Ann Intern Med 2004;141:352-9.

26. Nakagoe T, Sawai T, Tsuji T, Jibiki M, Ohbatake M, Nanashima A, et al. Surgical treatment and subsequent outcome of patients with carcinoma of the splenic flexure. Surg Today 2001;31:204-9.

27. Ogino S, Giannakis M. Immunoscore for (colorectal) cancer precision medicine. Lancet 2018;391:2084-6. 